\title{
Visual Outcome of Ocular Trauma Presenting in a Tertiary Care Hospital in Sahiwal
}

\author{
Ahmad Zeeshan Jamil ${ }^{1}$, Muhammad Luqman Ali Bahoo ${ }^{2}$ \\ ${ }^{1}$ Department of Ophthalmology, Sahiwal Medical College, Sahiwal \\ ${ }^{2}$ Department of Ophthalmology, Cornea Clinic, Shahida Islam Medical College, Lodhran
}

\begin{abstract}
Purpose: To find the visual outcome of ocular trauma presenting in a tertiary care hospital in Sahiwal.

Study Design: Descriptive observational study.

Place and Duration of Study: This study was conducted at District Headquarter Teaching Hospital affiliated with Sahiwal Medical College, Sahiwal from January 2016 to June 2019.

Methods: Four hundred and thirty five patients were included in the study by convenient sampling technique. Patient's age, gender, occupation, activity at the time of injury, nature of object causing trauma, duration of trauma, visual acuity at the time when patient reported to hospital, the time delay before coming to the hospital were recorded. Ocular injuries were classified according to Birmingham ocular trauma terminology. Detailed ocular examination was performed. Patients were managed and post-management visual acuity at three months was recorded.
\end{abstract}

Results: Mean age of patients was $29.07 \pm 12.53$ years. There were $219(50.3 \%)$ closed globe and $216(49.7 \%)$ open globe injuries. In $59(13.6 \%)$ cases, cause of injury was metal object. In $146(33.6 \%)$ cases injury was classified as contusion. In $164(37.7 \%)$ cases cornea was involved. Majority of the patients were male. Eightyseven patients presented within 1 day after trauma. In $154(35.4 \%)$ patients, visual acuity at the time of presentation was $6 / 12$ or better while in $171(39.3 \%)$ cases it was less than 6/60. Chi-square test was used to calculate the difference between pre and post-management visual acuity. This difference was statistically significant with $p$-value less than 0.05

Conclusion: Ocular trauma cases, if properly and timely managed, have a statistically significant increase in visual acuity.

Key Words: Blindness. Ocular trauma. Visual Acuity, contusion.

How to Cite this Article: Jamil AZ, Bahoo MLA. Visual Outcome of Ocular Trauma Presenting in a Tertiary Care Hospital in Sahiwal. Pak J Ophthalmol. 2020, 36 (2): 130-135.

Doi: $10.36351 /$ pjo.v36i2.987

Correspondence: Ahmad Zeeshan Jamil

Sahiwal Medical College

Sahiwal

Email: ahmadzeeshandr@yahoo.com

Received: January 21, 2020

Accepted: March 13, 2020

\section{INTRODUCTION}

Trauma to the eye and peri-orbital region can caused rastic consequences. Fortunately, most of the ocular trauma can be prevented. ${ }^{1}$ In fact, ocular trauma is one of the leading causes of blindness. ${ }^{2-4}$ Annually about $1,50,0000$ persons become blind because of ocular trauma. ${ }^{5}$ Patient's visit to the hospital as a result of eye injuries put a burden to already resource depleted 
health care system. ${ }^{6}$ Patients' sufferings from ocular trauma are not limited to the physical disabilities, but trauma also results in significant psychological and financial loss. ${ }^{7}$ Eye trauma limits a person's ability to work efficiently. Significant cosmetic disfigurement can happen after ocular injuries. As a result, the sufferer is depleted in terms of monetary resources and he is psychologically dejected. Consequences of ocular trauma can be long-lived and that can affect the whole family. Suffering incurred on the patient by the loss of vision is far more in magnitude than the loss of any other sense organ of the body. Eyes are vulnerable to get hurt during almost all kinds of daily life activates unless they are properly taken care of. One may get his/her eyes injured while playing, during daily home chores, at the time of work at the office, during travelling and due to assault. ${ }^{8}$ It is due to the anatomy of the eye that even minor trauma can cause significant damage to the eye. Ocular injuries can be occupationspecific. Most of the eye injuries occur with objects that persons deal most of the times. Every twentieth patient coming to an eye specialist for ocular treatment is a result of ocular trauma. ${ }^{9}$ Trauma can cause damage to the eye immediately or subsequent complications like infection or inflammation can lead to vision loss. The sequel of ocular trauma can cause significant ocular morbidity even after months or years. ${ }^{10}$ Selfmedication by the patients and intervention by an untrained health professional may lead to a suboptimal visual recovery in ocular trauma cases. By adopting safety-measure in situations when eyes can be injured, burden of ocular morbidity and irreversible blindness can be lessened. Appropriate management of ocular injuries by a trained health professional is of utmost importance to maximize visual recovery. ${ }^{11}$

There is scarcity of data in developing countries related to ocular injuries patterns, management protocols and visual outcomes. Because eye injury aetiology and pattern may vary from region to region, we want to know the pattern and characteristics of ocular trauma presenting to District Headquarter Teaching Hospital Sahiwal. We have tried to find out the visual outcome of the patients sustaining eye injuries. This will help in defining the characteristics of the ocular injuries in patients coming to our department. This can further help in designing and improving management strategies.

\section{METHODS}

This cross-sectional study was conducted at District Headquarter Teaching Hospital affiliated with Sahiwal Medical College, Sahiwal from January 2016 to June 2019. Following formula was used to calculate the sample size.

$\mathrm{S}=\mathrm{Z}^{2} \mathrm{p}(1-\mathrm{p}) / \mathrm{M}^{2}$

$\mathrm{S}$ is the sample size

$\mathrm{Z}$ is $\mathrm{Z}$ score its value is 1.96

$\mathrm{P}$ is the population proportion assumed to be $50 \%$ or 0.5

$\mathrm{M}$ is the margin of error that is taken $5 \%$ or 0.05

$$
\begin{aligned}
\mathrm{S} & =(1.96)^{2}(0.5)(1-0.5) / 0.05 \\
& =384.16 \\
& =384
\end{aligned}
$$

All patients of ocular trauma admitted in the eye department of District Headquarter Teaching Hospital Sahiwal were included in the study. Detailed information was shared with the patients and informed consent was taken. Patient's age, gender, occupation, activity at the time of injury, nature of object causing trauma, duration of trauma, visual acuity at the time of presentation, time delay before coming to the hospital were recorded.

To classify ocular trauma, Birmingham eye trauma terminology was adopted. Generally, eye injuries were classified into two broad categories namely open globe injuries and closed globe injuries. Closed globe trauma was again of two types; lamellar laceration and contusion. Laceration and rupture were two types of open globe injuries. Blunt injuries resulted in rupture of the globe and lacerations were caused by injuries with sharp objects. Penetration was a type of laceration in which globe wall was cut on one side only and the wound of entry was present. Perforation was a type of laceration in which globe wall was cut on both sides and wound of entry and wound of exit were present. Open globe injuries with intraocular foreign bodies were a sort of lacerations where one or more foreign bodies were present in the globe. ${ }^{12}$ Apart from injuries involving the globe, trauma to the eyelids and periorbital areas were also recorded. Patients were enquired about the time delay between the occurrence of injury and their report to the hospital. At the time of admission, patients' visual acuity was recorded. Slitlamp examination was performed in all patients. Bscan ultrasound examination was performed to find out 
intraocular foreign bodies and the status of the posterior segment in cases of media opacity. X-ray or CT scan imaging was performed when or where indicated. Patients were managed accordingly. Followup visits were done at the scheduled interval. At each visit, a detailed examination of the patient was performed. Visual acuity at three months post-trauma was recorded. Patients' information was collected by special proforma. Statistical package for social sciences version 23 was used to do statistical analysis. For age of the patients, mean and standard deviation was used. For gender, types of injuries, etiological agents, involved structures, visual acuity at the time of presentation and post-management visual acuity, frequencies and percentages were calculated. ChiSquare test was used to calculate the difference between pre and post-management visual acuity. Probability was considered significant at or below the value of 0.05 .

\section{RESULTS}

Four hundred and thirty-five patients with ocular trauma were included in the current study. Male patients were $301(69.2 \%)$ and female patients were $134(30.8 \%)$. Mean age of the patients was $29.07 \pm$ 12.53 years. Table number 1 describes the case distribution with respect to gender and age. Two hundred and sixteen $(49.7 \%)$ patients had open globe injuries and $219(50.3 \%)$ patients had closed globe injuries. Most of the injuries occurred due to trauma

Table 1: Distribution of cases according to gender and age.

\begin{tabular}{llll}
\hline Gender of Patient & Number & Mean & Std. Deviation \\
\hline Male & 301 & 28.41 & 14.03 \\
Female & 134 & 30.54 & 8.04 \\
Total & 435 & 29.07 & 12.52 \\
\hline
\end{tabular}

with a metallic piece. Table number 2 shows the percentage of trauma with different objects. There were $65(14.9 \%)$ cases of penetration, $30(6.9 \%)$ cases of intraocular foreign bodies, $146(33.6 \%)$ cases of contusion, $108(24.8 \%)$ cases of rupture, 74 (17.0\%) cases of lamellar laceration and $12(2.8 \%)$ cases of globe perforation. Corneal injuries were seen in 164 (37.7\%) cases, sclera was involved in $49(11.3 \%)$ cases, the corneo-scleral injury was present in 136 $(31.3 \%)$ cases and adnexa injuries were present in 30 $(6.9 \%)$ cases. There was lid tear in $14(3.2 \%)$ cases, blow out fracture was seen in $2(0.5 \%)$ cases and periocular swelling was present in $19(4.4 \%)$ cases. In $21(4.8 \%)$ cases, posterior segment was a major site of injury. Hyphema was seen in $206(47.4 \%)$ cases, lens damage occurred in $182(41.8 \%)$ cases, vitreous haemorrhage was encountered in $112(25.7 \%)$ cases, retinal tear was seen in $14(3.2 \%)$ cases, retinal detachment was encountered in $31(7.1 \%)$ cases, commotio retinae was found in $25(5.7 \%)$ cases. Optic nerve swelling was present in $37(8.5 \%)$ cases. In 133 $(30.6 \%)$ cases intraocular contents were prolapsed.

Table 2: Distribution of cases according to aetiology.

\begin{tabular}{lcc}
\hline Causative Agent & Number & Percent \\
\hline Metal & 59 & 13.6 \\
Wood/Vegetable matter & 56 & 12.9 \\
Stone/Brick & 29 & 6.7 \\
Road Accident & 40 & 9.2 \\
Wielding arc & 11 & 2.5 \\
Acid (chemical) & 20 & 4.6 \\
Alkali (chemical) & 20 & 4.6 \\
Superglue (chemical) & 17 & 3.9 \\
Oil/Cooking Oil & 16 & 3.7 \\
Hand trauma & 21 & 4.8 \\
Fall & 12 & 2.8 \\
Firework & 19 & 4.4 \\
Fire & 10 & 2.3 \\
Pellet gun & 30 & 6.9 \\
Plant sap & 8 & 1.8 \\
Insect fall/bite & 15 & 3.4 \\
Animal attack & 12 & 2.8 \\
Glass & 23 & 5.3 \\
Plastic Scale & 13 & 3.0 \\
Tennis Ball & 4 & .9 \\
\hline Total & 435 & 100.0 \\
\hline
\end{tabular}

Table 3: Distribution of cases according to the place of injury.

\begin{tabular}{lcc}
\hline Place of Occurrence & Number & Percentage \\
\hline Workplace & 93 & 21.4 \\
Road traffic accident & 50 & 11.5 \\
Home & 103 & 23.7 \\
Sports & 34 & 7.8 \\
Assault & 64 & 14.7 \\
At school & 27 & 6.2 \\
Outdoor & 64 & 14.7 \\
\hline Total & 435 & 100.0 \\
\hline
\end{tabular}

Table number 3 shows the percentage of injuries occurred in different settings.6/12 or better visual acuity was noted in $154(35.4 \%)$ cases at the time of presentation to hospital, presenting visual acuity between 6/18 and 6/60 was recorded in 110 (25.3\%) 
cases and presenting visual acuity less than $6 / 60$ was seen in 171 (39.3\%). Comparison of visual acuity at the time of presentation and post-management visual acuity in ocular trauma patients is shown in figure number 1. Table number 4 gives the distribution of cases according to time to presentation in the hospital. Post management visual acuity was $6 / 12$ or better in $211(48.5 \%)$ cases, $6 / 18$ to $6 / 60$ in $105(24.1 \%)$ cases and less than 6/60 in $119(27.4 \%)$ cases. Chi-Square test was used to calculate the difference between pre and post-management visual acuity. The difference was statistically significant with a p-value of 0.000 .

Table 4: Distribution of cases according to time to presentation in Hospital.

\begin{tabular}{lcc}
\hline Time of Presentation & Number & Percent \\
\hline Within 1 day & 379 & 87.1 \\
Within 1 week & 45 & 10.3 \\
After 1 week & 11 & 2.5 \\
\hline Total & 435 & 100.0 \\
\hline
\end{tabular}

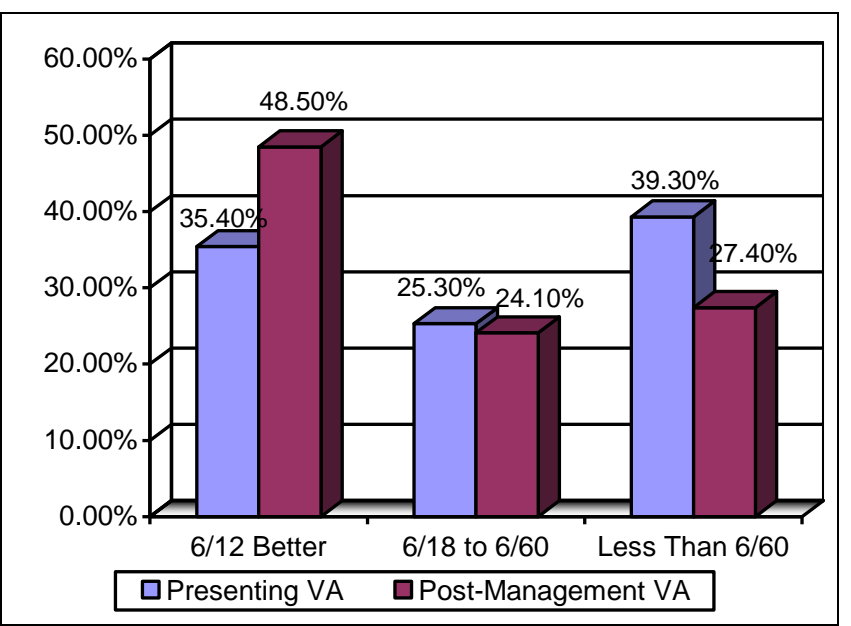

Fig. 1: Comparison of Presenting and Post-management Visual Acuity in Ocular Trauma Patients.

\section{DISCUSSION}

Ocular injuries are among the leading causes of blindness. ${ }^{13}$ Four hundred and thirty-five patients sustaining ocular injuries were studied in this hospitalbased study. Patients' mean age was $29.07 \pm 12.53$ years. Results of study in Dhulikhel Hospital of Kathmandu are in close approximation with the results of our study. In their study, most of the ocular injuries occurred in the age group of $21-30$ years. ${ }^{14}$ In the study of Godar and co-authors, most of the ocular injuries were encountered in the age group between 21 to 29 years. Young people are engaged in an active lifestyle and they tend to take risks that may be the reason for an increased number of ocular trauma seen in young people. As young people are bread winners for the family, so disabilities incurred as a result of ocular trauma has a great impact on the whole family finances and quality of life.

In our hospital-based study $301(69.2 \%)$ male and $134(30.8 \%)$ female patients were included. Work performed by Sengupta and co-authors also found that male patients are more prone to ocular injuries as compared to female patients. ${ }^{15}$ Males are busy in outdoor activities. That may be the reason for males to be involved in ocular injuries more often than females. ${ }^{16}$

In the present study, 219 (50.3\%) patients presented with closed globe injuries and 216 (49.7\%) patients came with open globe injuries. Contusion was the most common closed globe trauma. While globe rupture was the most common finding in patients coming with open globe trauma. Present study findings are consistent with the results of other studies. ${ }^{14,15}$ Majority of injuries were sustained due to trauma with a piece of metal and cornea was injured in most of the cases. These findings are also similar to the findings depicted in other studies. ${ }^{17}$

It is surprising to note that in our study most of the trauma was encountered in home settings. Home is considered the most secure place but paradoxically lots of trauma to the eye was seen there. Our study showed similar results as were recorded by Shaeri and coauthors where the majority of ocular trauma occurred in homes. ${ }^{18}$ Our findings are not in accord with the results of other researchers' studies that showed workplaces and road traffic accidents were the most common settings where the majority of ocular trauma occurred. ${ }^{13,15,19}$ An explanation for such a high number of ocular injuries at home setting may be the inadequate safety measure taken at home. Inadequate knowledge of safety measures or lack of access to safety goggles and eye shields may be the contributing factors. Children and women who spend most of their times in homes sustain most of the injuries at home settings. Education regarding eye safety and provision of eye protective devices should be readily available to masses. Use of eye protective devices is as much important during household activities as is important during work or during driving.

In the current study $379(87.1 \%)$ cases presented within one day after ocular trauma, $45(10.3 \%)$ cases presented to hospital within 7 days after ocular trauma 
and $11(2.5 \%)$ cases reported to the hospital after 7 days of sustaining ocular trauma. Findings of our study are similar to the findings of the study of Godar and coauthors. $^{13}$

In the current study $171 \quad(39.3 \%)$ patients presented with visual acuity of less than 6/60. Sengupta and co-authors conducted a study on ocular trauma where they found presenting visual acuity was less than $3 / 60$ in majority of the cases ${ }^{15}$. While Iqbal and the co-authors conducted study on ocular trauma patients and found good visual acuity in most of the cases. ${ }^{19}$ This difference in post-trauma presenting visual acuity may be due to selection bias. In the present study, patients with severe ocular trauma who required hospital admission were included. Minor trauma patients who required management on outdoor basis were not included in the current study.

Post management visual acuity of $6 / 12$ or better was reported in 211 (48.5\%), 6/18 to 6/60 was reported in $105(24.1 \%)$ patients and less than 6/60 was noted in $119(27.4 \%)$ cases. Probability (p) value was calculated for presenting visual acuity and postmanagement visual acuity difference. This difference was found to be statistically significant at a value of 0.000 .

In a study by Kuhn and co-authors, postmanagement visual acuity was $6 / 12$ or better in majority of the cases of blunt trauma while those having trauma with sharp objects had poor postmanagement visual acuity. ${ }^{12}$ In another study done by Cillino and colleagues post-management final bestcorrected visual acuity after ocular trauma was equal to or better than $6 / 12$ in $48.3 \%$ cases, from $6 / 12$ to $6 / 60$ final visual acuity was seen in $30.2 \%$ cases and less than $6 / 60$ final visual acuity was found in $15.5 \%$ cases. ${ }^{20}$ Our results are comparable to the results of their study.

The limitation of our study is the sample size and the selection of cases. In our study, patients who sustained major trauma to the ocular structures and required hospital admission were included. Patients with minor trauma not requiring hospital admission and who refused hospital admission were not included in the study. Moreover, our study is not the true representative of the ocular trauma as patients from high socioeconomic strata do not go to the public sector hospital.

The results of our study can be used to devise strategies for patient counselling to adopt ocular safety and to modify our management protocol and arrange resources at district level for early and better management.

\section{CONCLUSION}

Ocular trauma cases, if properly and timely managed, have a statistically significant increase in visual acuity.

\section{Ethical Approval}

The study was approved by the Institutional review board/Ethical review board.

\section{Conflict of Interest}

Authors declared no conflict of interest

\section{REFERENCES}

1. Bahoo MLA, Jamil AZ. Types of ocular surface foreign bodies and their correlation with location in the eye. Pak J Ophthalmol. 2018; 34 (1): 25-29.

2. Jan S, Khan S, Khan MT, Iqbal A, Muhammad S. Ocular emergencies. J Coll Physici. 2004; 14: 333-336.

3. Guerra Garcia RA, Garcia D, Martinez FE, Garbey YE, Martinez RR. The Cuban ocular trauma registry. J Clin Exp Ophthalmol. 2013; 4 (2): 276.

4. Negral AD, Thylefors B. The global impact of eye injuries. Ophthalmic Epidemiol. 1998; 5: 143-169.

5. Tsedeke A, Yeshigeta G, Fessehaye A. A 2 year review of ocular trauma in Jimma University specialized hospital. Ethiop J Health Sci. 2009; 19: 6774.

6. Babar TF, Khan MN, Jan SU, Shah SA, Zaman M, Khan MD. Frequency and causes of bilateral ocular trauma. J Coll Physici. 2007; 17: 679-682.

7. Bahoo MLA, Jamil AZ, Khalid MS. Ocular surface foreign bodies and their association with profession. Pak J Med Health Sci. 2018; 12 (2): 495-698.

8. Khatry SK, Lewis AE, Schein OD, Thapa MD, Pradhan EK, Katz J. The epidemiology of ocular trauma in rural Nepal. Br J Ophthalmol. 2004; 88: 456460.

9. Magarakis M, Mundinger GS, Kelamis JA, Dorafshar AH, Bojovic B, Rodriguez ED. Ocular injury, visual impairment and blindness associated with facial fractures: a systematic literature review. Plast Reconstr Surg. 2012; 129: 227-233.

10. Bowling, B. Kanski's clinical ophthalmology a systematic approach, 2015; Sydney: Saunders.

11. Pandita A, Merriman M. Ocular trauma epidemiology: 10-year retrospective study. N Z Med J. 2012; 125: 61-69. 
12. Kuhn F, Morris R, Witherspoon D, Heimann K, Jeffers JB, Treister G. A standardized classification of ocular trauma. Ophthalmology, 1996; 103: 204-213.

13. Godar ST, Kaini KR, Amatya P, Joshi K, Singh L. Magnitude of ocular trauma in a tertiary care hospital of western Nepal. Nepal J Med Sci. 2013; 2 (2): 140-143.

14. Sthapit PR, Marasini S, Khoju U, Thapa G, Nepal BP. Ocular Trauma in patients presenting to Dhulikhel Hospital. Kathmandu Univ Med J. 2011; 33: 54-57.

15. Sengupta P, Mazumdar M, Gyatsho J. Epidemiology of ocular trauma cases presenting to a tertiary care hospital in a rural area in West Bengal, India over a period of 2 years. IOSR-JDMS. 2016; 15: 92-97.

16. Bahoo MLA, Jamil AZ, Khalid MS. Ocular surface foreign bodies and their association with profession. Pak J Med Health Sci. 2018; 12: 495-498.

17. Oum BS, Lee JS, Han YS. Clinical features of Ocular Trauma in Emergency Department. Korean J Ophthalmol. 2004; 18: 70-78.

18. Shaeri M, Moravveji A, Fazel MR, Jeddi FR. Status of ocular trauma in hospitalized patients in Kashan, 2011: As a sample of industrial city. Chin J Traumatol. 2016 Dec 1; 19 (6): 326-329.
19. Iqbal Y, Khan QA, Zia S, Malik A. Frequency and characteristics of ocular trauma in Gilgit, Pakistan. J Islam Int Med Coll. 2016; 11: 157-162.

20. Cillino S, Casuccio A, Di Pace F, Pillitteri F, Cillino G. A five-year retrospective study of the epidemiological characteristics and visual outcomes of patients hospitalized for ocular trauma in a Mediterranean area. BMC Ophthalmology, 2008; 8: 6. Doi: 10.1186/1471-2415-8-6.

\section{Authors' Designation and Contribution}

Dr Ahmad Zeeshan Jamil; Associate Professor: Concept and design of study. Statistical analysis, manuscript writing and interpretation of data.

Dr. Muhammad Luqman Ali Bahoo; Associate Professor: Literature search, drafting of the article, critical revision and proof reading. 